\section{One-Component Approximation for Binary Diblock Copolymer Blends}

\section{W. Matsen* and F. S. Bates}

Department of Chemical Engineering and Materials Science, University of Minnesota, Minneapolis, Minnesota 55455

Received June 16, 1995

Revised Manuscript Received August 4, 1995

Materials science is a field in which researchers strive not only to understand material properties but also to develop the predictive power to produce new materials with desired properties. Metallurgy is one subfield which has been rather successful in this regard. From a limited number of atomic species, a rich complement of alloys have been developed through blending, producing a wide range of useful properties. Prediction of phase behavior in atomic mixtures is aided by the Hume-Rothery rules ${ }^{1}$ which help anticipate miscibility of components and resulting crystal structures. Naturally, it would be desirable to obtain similar guidelines for blending block copolymers, and that is the goal of this study.

This communication examines binary blends of $\mathrm{AB}$ diblock copolymers where both molecules have the same number of segments, $N$, but unequal A block fractions, $f_{1}$ and $f_{2}$. The $\mathrm{A}$ and $\mathrm{B}$ monomers are taken to be conformationally symmetric (i.e., equal statistical segment lengths), and immiscibility between them is controlled through the usual Flory-Huggins parameter, $\chi$. Using self-consistent field theory (SCFT), we examine the "classical" block copolymer phases, lamellae (L), hexagonally $(\mathrm{H})$ packed cylinders, body-centered cubic (C) spheres, and disordered (D) phases. Our study is similar to that in ref 2 except here coexisting phases are properly accounted for. To determine coexistence regions, we use the grand-canonical SCFT approach described in ref 5.

Although our calculation is performed in the grandcanonical ensemble where a chemical potential controls the volume fraction of the second copolymer species, $\phi$, our results are presented in terms of the canonical coordinates, $\chi N, f_{1}, f_{2}$, and $\phi$. With four independent coordinates, we rely on two-dimensional slices to describe phase behavior. Since the phase diagram depends least on $\chi N$, we fix that quantity at 20 and describe how the $f_{1}-f_{2}$ plane evolves with $\phi$. When the volume fraction of the second diblock, $\phi$, is zero, the transitions, $\mathrm{D} \leftrightarrow \mathrm{C} \leftrightarrow \mathrm{H} \rightarrow \mathrm{L} \rightarrow \mathrm{H} \rightarrow \mathrm{C} \leftrightarrow \mathrm{D}$, are independent of $f_{2}$ and occur at $f_{1}=0.210,0.243,0.363$, $0.637,0.757$, and 0.790 , respectively. Because this limit corresponds to a one-component melt, there are no biphasic regions and the $f_{1}-f_{2}$ plane remains similar to that in ref 2 except for slight differences due to the unitcell approximation they used. As the second diblock is added and $\phi$ increases, phase boundaries shift and biphasic regions appear between single-phase regions. Figures 1 and 2 show the phase boundaries for $\phi=0.25$ and 0.50 , respectively. As $\phi$ increases, $f_{2}$ has a greater influence on the phase behavior. Because of the symmetry associated with interchanging the labels, 1 and 2 , it is not necessary to consider $\phi>0.50$. We note that calculations at $\chi N=40$ show results similar to those above; these will be reported in a future, comprehensive report.

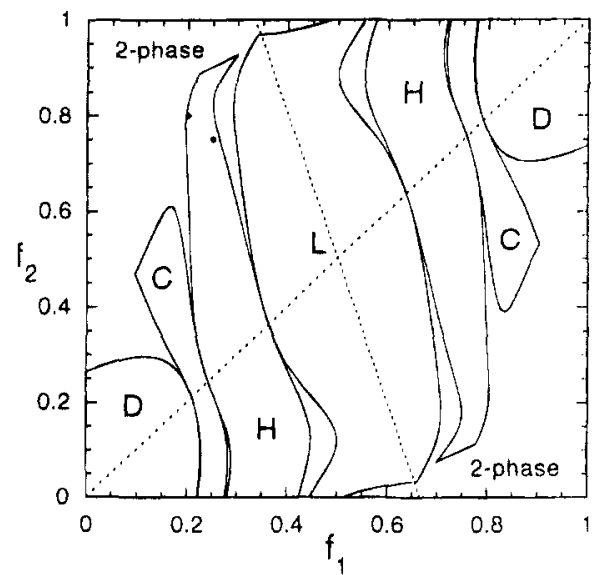

Figure 1. Phase diagram for $\chi N=20$ and $\phi=0.25$ (i.e., $75 \%$ of the diblocks have an A block fraction $f_{1}$, and $25 \%$ have an $\mathrm{A}$ block fraction $f_{2}$ ). Single-phase regions are labeled L (lamellar), $\mathrm{H}$ (hexagonal cylinders), $\mathrm{C}$ (bcc spheres), and D (disordered). The one-component diagonal and the $f=0.5$ line are shown with dotted lines. The diamonds denote points which also occur in Figures 3 and 4.

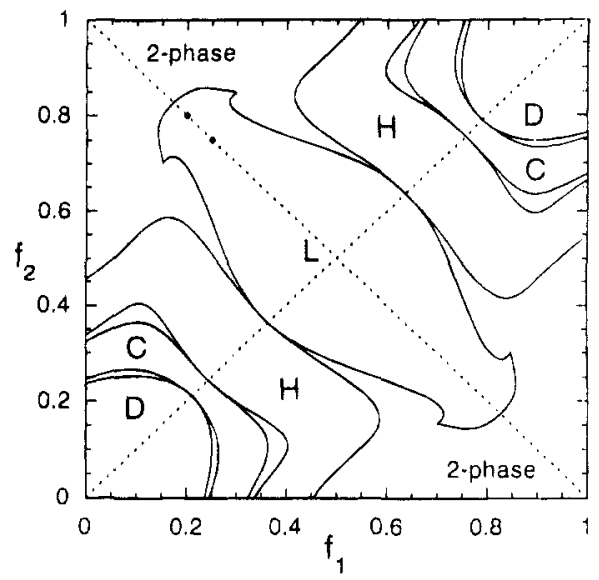

Figure 2. Phase diagram similar to that in Figure 1 but for $\chi N=20$ and $\phi=0.50$.

Along the diagonal, $f_{1}=f_{2}$, the system is a onecomponent melt. Consequently, the influence of $\phi$ on phase behavior and the size of biphasic regions become asymptotically small as this line is approached. As $\phi$ varies, phase boundaries twist about fixed points along the diagonal. As noted in ref 2 , they twist in such a way that the slopes of the transitions at the fixed points follow lines of constant A monomer volume fraction, $f$ $\equiv(1-\phi) f_{1}+\phi f_{2}$. This result is derived from the fact that along this diagonal, partial derivates of the Helmholtz free energy, $A\left(\chi N, f_{1}, f_{2}, \phi\right)$, with respect to $f_{1}, f_{2}$, and $\phi$ are proportional to $1-\phi, \phi$, and 0 , respectively. Consequently, the derivative of $A$ is zero along directions where $\chi N$ and $f$ remain constant. Because biphasic regions are negligible and phase behavior is dictated primarily by $\chi N$ and $f$, the phase behavior of the blend approximately maps onto that of the pure diblock melt. Not only does this make it relatively easy to predict the structure a blend will exhibit, it provides a powerful way of locating phase boundaries in pure diblock melts. By synthesizing two diblocks, one on each side of a transition, blending permits the accurate determination of $f$ at the transition. This procedure was used in ref 3 , and in a forthcoming paper, ${ }^{4}$ further experimental and theoretical justification for it will be presented.

When the diblocks start to differ by too much (i.e., $\mid f_{1}$ $-f_{2} \mid \gtrsim 0.1$ ), transitions begin to signficantly deviate 


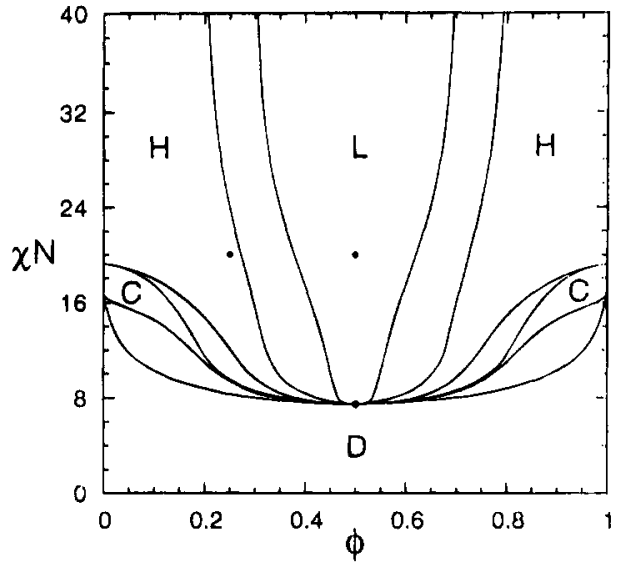

Figure 3. Phase diagram for $f_{1}=1-f_{2}=0.25$, where $\phi$ is the volume fraction of component 2 . The dot denotes the critical point, and diamonds denote points occurring in Figures 1 and 2.

from lines of constant $f$ and biphasic windows between single-phase regions become large. Still, the onecomponent approximation can be used to qualitatively predict the phase behavior. However, if either $f_{1}$ or $f_{2}$ becomes close to 0 or 1 , this approximation completely breaks down. This is because the junctions of highly asymmetric diblocks are no longer constrained to the internal interfaces of a microstructure. These copolymers may continue to reside in the structure, causing its dimensions to swell, 2,5 but often they will phase separate into a disordered phase. Reference 2 did not allow for this possibility, and consequently, they predict swollen phases which are actually unstable with respect to phase separation. We note that these swollen phases do exist at compositions where the volume fraction of the highly asymmetric diblock is small.

The biphasic regions in Figures 1 and 2 warrant some discussion. They are complicated by the fact that tie lines are parallel to the $\phi$ axis and hence orthogonal to the $f_{1}-f_{2}$ plane. Therefore, these diagrams do not provide a clear indication of which phases are coexisting at a particular point in the two-phase region. In principle, this can be determined by knowing how the $f_{1}-f_{2}$ plane evolves with $\phi$. Near the single-component diagonal a relatively simple picture emerges. When crossing between two adjacent single-phase regions, a region is traversed where both phases coexist. On the other hand, leaving a single-phase region toward an edge of the diagram, where one of the diblocks becomes very asymmetric, often produces coexistence with a disordered phase. Where these different coexistence regions meet, triple points occur, producing kinks in the binodals.

To illustrate what happens as the one-component approximation breaks down, we focus on pairs of complementary diblocks, $f_{1}=1-f_{2}$. Over the range $0.3<f_{1}<0.7$, the approximation works well, and plotting phase transitions in terms of $\chi N$ and $f$ produces a phase diagram similar to that of the pure diblock system. The phase diagram for $f_{1}=0.25$, where the biphasic windows have become more substantial, is illustrated in Figure 3. Aside from the biphasic windows, the topology of the phase diagram is the same as for the pure diblock, where the ordered phases extend down to a single critical point. When $f_{1}$ is reduced to 0.20 , this is no longer the situation, as shown in Figure 4. Because the diblocks resemble homopolymers, there is now an Ising-type critical point, and as $\chi N$ increases

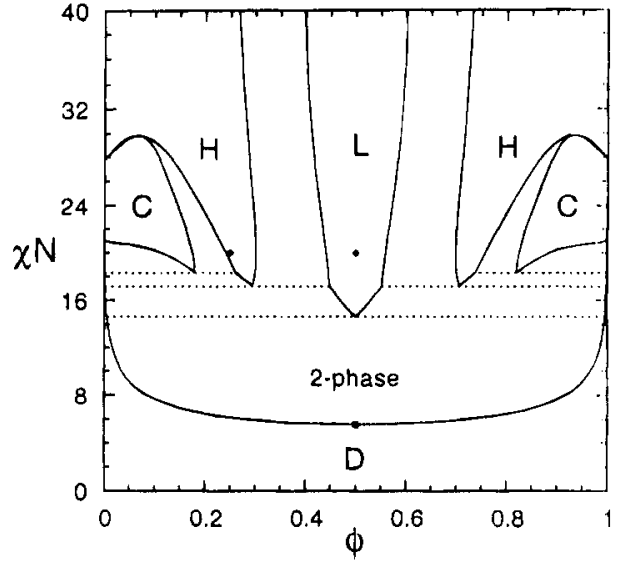

Figure 4. Phase diagram similar to that in Figure 3 but for $f_{1}=1-f_{2}=0.20$. Dotted lines denote triple lines.

from the disordered phase, the copolymers initially phase separate. Not until $\chi N=14.635$ do the diblocks mix to form a single microstructure.

References 6 and 7 describe experiments conducted on blends of complementary cylinder-forming diblocks similar to those in Figure 3. Both studies find that 50: 50 blends produce a L structure as opposed to two coexisting $\mathrm{H}$ phases as might be expected. The present calculation is the first to substantiate this result theoretically. Although ref 2 predicted a L phase, they did not address the possibility of coexisting $\mathrm{H}$ phases. This is also the case for simulations in ref 6 , because their small system prevents coexistence. ${ }^{8}$ Moreover, there would be significant finite-size effects for ordered phases in ref 6 , since the period is required to be commensurate with the system size.

On the basis of previous work, ${ }^{5}$ there are stable structures in addition to those considered here. Reasonable speculations can be made regarding how they would alter the behavior described above. First, the bccarranged spheres in the $\mathrm{C}$ phase will switch to a closepacked arrangement when one of the diblocks becomes sufficiently asymmetric. Secondly, a window of complex phases would occur between the $\mathrm{L}$ and $\mathrm{H}$ ones. Along the single-component diagonal, the complex phase would be a bicontinuous cubic one with $I a \overline{3} d$ symmetry. Moving off the diagonal, other complex phases such as a hexagonally-perforated lamellar phase and the bicontinuous cubic phase with $P n \overline{3} m$ symmetry could occur. It is likely that there also will be other complex phases in this region which cannot be anticipated. Because the SCFT requires the symmetry of these structures to be known a priori, this possibility could not be accounted for. This is, in part, why complex phases are omitted from our calculation. It makes sense to await further experiments before considering them. Our calculation will nevertheless provide an indication of where experiments should search for them.

In summary, we have examined the phase behavior for blends of two $A B$ diblocks with equal polymerization indexes. Our results illustrate that for closely matched diblocks, the phase behavior is to a good approximation dictated by $\chi N$ and $f \equiv(1-\phi) f_{1}+\phi f_{2}$. This results in a one-component approximation that maps the phase behavior of the blend onto that of the pure diblock melt. When the dissimilarity between diblocks increases, large biphasic windows occur between single-phase regions, but as long as neither diblock becomes too asymmetric, the one-component approximation still qualitatively predicts the phase behavior of the blend. 
Acknowledgment. This work has been supported by NSF (DMR 94-05101) and by the Minnesota Supercomputer Institute.

\section{References and Notes}

(1) Smallman, R. E.; Hume-Rothery, W.; Haworth, C. W. The Structure of Metals and Alloys; Institute of Metals, London, 1988.

(2) Shi, A.-C.; Noolandi, J. Macromolecules 1995, $28,3103$.

(3) Zhao, J.; Majumdar, B.; Schulz, M. F.; Bates, F. S.; Almdal, K.; Mortensen, K.; Hajduk, D. A.; Gruner, S. M. Macromolecules, in press.

(4) Schulz, M. F.; Khandpur, A. K.; Matsen, M. W.; Bates, F.
S.; Almdal, K.; Mortensen, K.; Hajduk, D. A.; Gruner, S. M. Manuscript in preparation.

(5) Matsen, M. W. Phys. Rev. Lett. 1995, 74, 4225; Macromolecules 1995, 28, 5765 .

(6) Vilesov, A. D.; Floudas, G.; Tadeusz, P.; Melenevskaya, E. Yu.; Birshtein, T. M.; Lyatskaya, Y. V. Macromol. Chem. Phys. 1994, 195, 2317.

(7) Koizumi, S.; Hasegawa, H.; Hashimoto, T. Macromolecules 1994, 27,4371 .

(8) To examine coexistence in the canonical ensemble using Monte Carlo, the system size must be large enough that resulting interfacial tension is negligible in comparison to the bulk free energy.

MA950854H 